\title{
Computed Tomography-based determinants for predicting death and ICU requirement in patients suffering COVID-19
}

Marzie Dehgan ( $\square$ Marzi.dehgan@gmail.com )

Iran University of Medical Sciences

Nasrin Khalesi

iran university of medical sciences

Masumeh Akbari

IUMS: Iran University of Medical Sciences

Rogheyeh Fallah

IUMS: Iran University of Medical Sciences

Neda Hosseininezhad

IUMS: Iran University of Medical Sciences

\section{Research}

Keywords: Coronavirus Disease 2019, COVID -19, Computed Tomography, Intensive Care Units, lung

Posted Date: October 28th, 2020

DOl: https://doi.org/10.21203/rs.3.rs-97828/v1

License: (c) (1) This work is licensed under a Creative Commons Attribution 4.0 International License. Read Full License 


\section{Abstract}

Background: What has received special attention in recent months is the use of a combination of clinical findings, laboratory markers, and, in addition, the findings of lung Computed Tomography (CT) scan in the design and delivery of risk scoring systems for Coronavirus Disease 2019 (COVID -19) patients. The present study aimed to determine main lung CT-related correlates of disease severity (Intensive Care Units (ICU) requiring) as well as death in COVID -19 patients.

Methods: This cross-sectional study was performed on 515 consecutive patients with definitive diagnosis of COVID-19 admitted to one of the COVID -19 referral hospitals in Tehran. All patients' information was collected through a review of their archives. All patients were evaluated by CT scan of the lungs.

Results: The mean follow-up of patients from the time of admission was $10.85 \pm 6.11$ days between 1 and 30 days. During this period, a total of $29.1 \%$ were admitted to the ICU. Also, the mortality rate of patients was equal to $28.2 \%$. According to multivariable logistic regression model with the presence of deathrelated correlates, crazy paving pattern, diffuse distribution of lesions, CT Severity Score (CTSS) score $>12$, the presence of plural effusion or emphysema were the main determinants of COVID -19 related death and should be considered for presenting new scoring system for predicting death following COVID -19 disease. In similar model, CTSS score $>12$ along with the presence of plural effusion, emphysema, or pulmonary hypertension were the main determinants of requiring ICU admission.

Conclusion: The CT score higher than 12 along with observing the pattern of diffuse distribution of lesions especially accompanied with emphysema, pleural effusion or pulmonary hypertension can predict patient mortality or will determine the need for hospitalization in the ICU.

\section{Introduction}

By the end of September 2020, more than 35 million people had been infected with Coronavirus Disease 2019 (COVID-19), leading more than one million deaths worldwide certainly due to the onset of the second and even third waves of disease in some countries [1]. Common clinical manifestations of the disease include acute respiratory syndrome with fever and dry cough, dyspnea, fatigue, and myalgia. About 15 to $20 \%$ of patients also experience severe illness, which is associated with mortality of about 2 to $3 \%$ [2-4]. In the absence of vaccine or specific treatment available to date, the diagnosis of the disease as quickly and accurately as possible and its severity are essential that can prevent further progression of the disease. However, pharyngeal sampling and using Polymerase Chain Reaction (PCR) technique is still the final reference diagnosis, but it is time consuming and the sensitivity of this test is estimated at about 60 to $70 \%$ due to the quality of sampling and the rate of virus replication in the corresponding position [5, 6]. In this regard, Computed Tomography (CT) scan is a rapid and accessible tool for diagnosing and tracking the severity of pneumonia caused by COVID-19 disease [7, 8]. Due to the high influx of patients to emergency and hospital centers, it is necessary to triage as soon as possible the patients who refer and have symptoms suspected of having the disease. According to recent guidelines provided by the 
World Health Organization and associations related to the prevention and control of the disease, the use of CT scan of the chest for all patients with manifestations such as dyspnea, polypnea and reduced arterial oxygen saturation is necessary to differentiate between the two groups of patients requires hospitalization or only needs outpatient treatment and follow-up, which should be synchronized with PCR [9]. The use of CT scan due to its high sensitivity, low radiation dose and no need for contrast, has been very useful in screening symptomatic patients, but the presence of low specificity and some atypical changes similar to other pneumonias such as influenza and or adenovirus infections limit its use. Therefore, lung CT cannot be considered as a definitive method of diagnosis for the disease. However, due to the high sensitivity of this tool, it is one of the best methods for estimating the severity of the disease in patients with a definitive diagnosis of COVID-19. In this regard, some algorithms for estimating and predicting disease severity based on CT findings have been proposed. But despite the presentation of all these classification systems, the presentation of a system with the highest sensitivity and specificity in distinguishing mild from severe clinical involvement is still under discussion and study. The present study aimed to determine main lung CT-related correlates of disease severity (Intensive Care Units (ICU) requiring) as well as death in COVID-19 patients.

\section{Methods}

This cross-sectional study was performed on 515 consecutive patients with definitive diagnosis of COVID-19 admitted to one of the COVID - 19 referral hospitals in Tehran, Iran between April and September 2020. All patients' information was collected through a review of their archives, and therefore patients with incomplete information about demographic information, CT findings, and disease prognosis were excluded from the study. Definitive diagnosis was made based on a specific COVID-19 diagnostic kit and RT-PCR of the virus genome in the laboratory (isolation of SARSCoV-2 or at least two positive tests of the virus genomic test). All patients were evaluated by CT scan of the lungs. Chest CT scans were performed with CT system Siemens Healthineers, Germany in supine position. The imaging parameters were as follows: tube voltage $100 \mathrm{kVp}$, current $80 \mathrm{mAs}$, pitch $=1.5 \mathrm{~mm}$, thickness $1.5 \mathrm{~mm}$. The maximum time between the initial clinical evaluation and CT imaging of patients was one day. The whole image was interpreted by two radiologists who were unaware of each other's interpretation. What was examined in the CT images included the following features: 1) distribution of lesions (peripheral, Axial, bronchovascular perineum, diffuse, anterior, and pericardial), 2) types of lesions (Grand Glass opacities, consolidation, crazy paving pattern, reverse halo sign, centrilobular air space, bronchiectasis traction between lesions, and linear opacities), and 3) underlying pulmonary signs (bronchiectasis, emphysema, pleural effusion, and pulmonary hypertension). The CT severity score (CTSS) was also determined based on the scoring introduced by Kunwei et al. in this scoring system, all five lobes of both lungs are evaluated for the presence of disease-related lesions. Each lobe scores between 0 and 4 based on the percentage of lobar involvement in the form of $0(0 \%), 1$ (1 to $25 \%), 2$ (26 to $50 \%), 3$ (51 to $75 \%$ ) or 4 (76 to $100 \%$ ) [10]. The total score will be the sum of the scores for the five lobes of both lungs. The study endpoints were first disease-related death (occurring within 30 days of first admission) and second requiring ICU admission as an indicator for disease severity. 
For statistical analysis, results were presented as mean \pm standard deviation (SD) for quantitative variables and were summarized by frequency (percentage) for categorical variables. Continuous variables were compared using t test or Mann-Whitney test whenever the data did not appear to have normal distribution or when the assumption of equal variances was violated across the study groups. Categorical variables were, on the other hand, compared using chi-square test. To determine the value of quantitative parameters to predict disease outcome, the ROC curve analysis was employed. Also, for weighting each predictive parameter to achieve final scoring system, the multivariable logistic regression modeling was finally used. For the statistical analysis, the statistical software SPSS version 23.0 for windows (IBM, Armonk, New York) was used.

\section{Results}

A total of 515 patients were included in the study. The mean age of patients was $56.90 \pm 16.37$ years in the range of 21 to 89 years and in terms of sex distribution, $64.1 \%$ were male and $35.9 \%$ were female. In terms of underlying comorbidities, underlying heart disease in $18.4 \%$, history of lung disease in $13.6 \%$, history of diabetes in $7.8 \%$ and history of other diseases in $11.7 \%$. In terms of clinical manifestations, the most common symptoms were fever in $90.3 \%$, cough in $72.8 \%$, dyspnea in $39.8 \%$, myalgia in $25.2 \%$ and diarrhea in $4.9 \%$. The mean follow-up of patients from the time of admission was $10.85 \pm 6.11$ days between 1 and 30 days. In this follow-up, the average length of hospital stay was $5.78 \pm 4.27$ days between 1 and 18 days. During this period, a total of $29.1 \%$ were admitted to the ICU. Also, the mortality rate of patients was equal to $28.2 \%$.

First, at the time of admission, $8.7 \%$ of patients with CT scan were completely normal. Among other patients with pulmonary involvement, grand glass opacity in $59.2 \%$, consolidation in $29.1 \%$, crazy paving pattern in $7.8 \%$, reverse halo sign in $25.2 \%$, nodular profile in the form of centrilobular air space in $3.9 \%$, bronchiectasis traction between lesions in $32.0 \%$ and linear opacities in $46.6 \%$. Regarding the distribution of pulmonary involvement, peripheral distribution was found in $91.2 \%$, axial distribution in $56.3 \%$, peribronchovascular distribution in $20.4 \%$, diffuse distribution in $12.6 \%$, anterior distribution in $65.0 \%$ and pericardial distribution in $32.0 \%$. Other observable underlying pulmonary disorders included underlying bronchiectasis in $0.1 \%$, underlying emphysema in $5.8 \%$, pleural effusion in $61.1 \%$, and pulmonary hypertension in $13.6 \%$.

To determine the CTSS score, the mean score of RUL was $2.15 \pm 1.28$, the mean score of RML was $1.81 \pm$ 1.43 , the mean score of RLL was $2.56 \pm 1.29$, the mean score of LUL was $2.40 \pm 1.30$ and the mean score of LLL conflict was estimated to be $2.33 \pm 1.41$. In general, the mean total score of lung involvement was $10.90 \pm 5.83$.

Comparing pattern and distribution of lesions between survived and non-survived subgroups (Table 1) showed higher prevalence of crazy paving pattern in nun-survived group. Also, non-survived subgroup had higher prevalence of diffuse and pericardial distributions. In the two groups with and without death, the frequency of bronchiectasis was $3.4 \%$ and $0.0 \%(P=0.282)$, the frequency of emphysema was $13.8 \%$ 
and $2.7 \%(P=0.030)$, the frequency of pleural effusion was $82.8 \%$ and $52.7 \%(P=0.005)$, and pulmonary hypertension was $20.7 \%$ and $10.8 \%(P=0.210)$, respectively, indicating differences in the frequency of emphysema and pleural effusion. The mean CTSS score in the two groups was $14.27 \pm 5.09$ and $9.58 \pm$ 5.59 , respectively, which showed a significant difference between the two groups $(P<0.001)$. Based on the analysis of the area under the ROC curve, the evaluation of the CTSS score was considered to have a high value in predicting the occurrence of death (the area under the curve of $0.721,95 \%$ confidence interval between 0.616 and $0.826, P<0.001$ ). Accordingly, a cutoff value of 12.0 with a sensitivity of $79.0 \%$ and a specificity of $64.1 \%$ will be able to predict the occurrence of death in patients. 
Table 1

Comparing CT findings in survived and non-survived groups with Covid-19

\begin{tabular}{|llll|}
\hline Item & Survived group & Non-survived group & P value \\
\hline Form of lesion & & & \\
\hline Ground Glass Opacity & $72.4 \%$ & $54.1 \%$ & 0.088 \\
\hline Consolidation & $24.1 \%$ & $31.1 \%$ & 0.485 \\
\hline Crazy paving pattern & $17.2 \%$ & $4.1 \%$ & 0.020 \\
\hline Reverse halo sign & $18.2 \%$ & $28.4 \%$ & 0.430 \\
\hline Centrilobular air space & $0.0 \%$ & $5.4 \%$ & 0.202 \\
\hline Bronchiectasis traction & $44.8 \%$ & $27.0 \%$ & 0.082 \\
\hline Linear opacities & $44.8 \%$ & $47.4 \%$ & 0.787 \\
\hline Distribution of lesions & & & \\
\hline Peripheral & $100 \%$ & $87.8 \%$ & 0.059 \\
\hline Axial & $69.0 \%$ & $52.7 \%$ & 0.184 \\
\hline Peribronchovascular & $27.6 \%$ & $17.6 \%$ & 0.256 \\
\hline Diffuse & $27.6 \%$ & $6.8 \%$ & 0.004 \\
\hline Anterior & $79.3 \%$ & $59.5 \%$ & 0.057 \\
\hline Pericardial & $51.7 \%$ & $24.3 \%$ & 0.007 \\
\hline Underlying lung disorders & & $52.7 \%$ & 0.005 \\
\hline Bronchiectasis & $3.4 \%$ & $10.8 \%$ & 0.001 \\
\hline Emphysema & $13.8 \%$ & $9.58 \pm 5.59$ & 0.030 \\
\hline Pleural effusion & $82.8 \%$ & $2.7 \%$ & 0.005 \\
\hline Pulmonary hypertension & $20.7 \%$ & $14.27 \pm 5.09$ & \\
\hline Mean CTSS score & & & \\
\hline
\end{tabular}

Comparing form of lesions in the two groups with and without ICU admission (Table 2) showed no difference in the prevalence rate of ground glass opacity, consolidation, crazy paving pattern, reverse halo sign, centrilobular air space, or linear opacities. Those requiring ICU admission had higher rate of diffuse axial, anterior, and pericardial distributions as compared to non-ICU admission subgroup. In the two groups with and without ICU hospitalization, the frequency of bronchiectasis was equal to $0.0 \%$ and $1.4 \%$, the frequency of emphysema to $13.3 \%$ and $2.7 \%$ pleural effusion to $76.7 \%$ and $54.8 \%$ and pulmonary hypertension to $26.7 \%$ and $8.2 \%$, respectively. The differences were in the frequency of emphysema, 
pleural effusions and pulmonary hypertension. The mean CTSS score in the two groups with and without ICU admission was $13.53 \pm 4.36$ and $9.82 \pm 6.04$, respectively, which showed a significant difference between the two groups $(P=0.003)$. Based on the analysis of the area under the ROC curve, the evaluation of CTSS score was considered to have a high value in predicting ICU admission (area under the curve was $0.700,95 \%$ confidence interval between 0.594 and $0.803, P=0.002$ ). A cutoff point of 12.0 with a sensitivity of $73.3 \%$ and a specificity of $64.4 \%$ was able to predict the need for ICU hospitalization. According to multivariable logistic regression model with the presence of death-related correlates (Table 3), crazy paving pattern, diffuse distribution of lesions, CTSS score $>12$, the presence of plural effusion or emphysema were the main determinants of COVID-19 related death and should be considered for presenting new scoring system for predicting death following COVID-19 disease. In similar model (Table 4), CTSS score > 12 along with the presence of plural effusion, emphysema, or pulmonary hypertension were the main determinants of requiring ICU admission. 
Table 2

Comparing CT findings in groups requiring and no-requiring ICU

\begin{tabular}{|llll|}
\hline Item & Survived group & Non-survived group & P value \\
\hline Form of lesion & & & \\
\hline Ground Glass Opacity & $56.7 \%$ & $60.3 \%$ & 0.735 \\
\hline Consolidation & $40.0 \%$ & $24.7 \%$ & 0.119 \\
\hline Crazy paving pattern & $13.3 \%$ & $5.5 \%$ & 0.333 \\
\hline Reverse halo sign & $16.7 \%$ & $28.8 \%$ & 0.563 \\
\hline Centrilobular air space & $3.3 \%$ & $4.1 \%$ & 0.853 \\
\hline Bronchiectasis traction & $43.3 \%$ & $27.4 \%$ & 0.115 \\
\hline Linear opacities & $60.0 \%$ & $41.1 \%$ & 0.287 \\
\hline Distribution of lesions & & & \\
\hline Peripheral & $100 \%$ & $87.7 \%$ & 0.056 \\
\hline Axial & $80.0 \%$ & $47.9 \%$ & 0.003 \\
\hline Peribronchovascular & $26.7 \%$ & $17.8 \%$ & 0.311 \\
\hline Diffuse & $23.3 \%$ & $8.2 \%$ & 0.050 \\
\hline Anterior & $80.0 \%$ & $58.9 \%$ & 0.045 \\
\hline Pericardial & $53.3 \%$ & $23.3 \%$ & 0.003 \\
\hline Underlying lung disorders & & & 0.037 \\
\hline Bronchiectasis & $0.0 \%$ & $5.7 \%$ & 0.003 \\
\hline Emphysema & $13.3 \%$ & $9.82 \pm 6.04$ & \\
\hline Pleural effusion & $76.7 \%$ & & 0.039 \\
\hline Pulmonary hypertension & $26.7 \%$ & $1.4 \%$ & \\
\hline Mean CTSS score & $13.53 \pm 4.36$ & & \\
\hline
\end{tabular}


Table 3

Multivariable logistic regression model to determine the main CT-based correlates of death in Covid-19 patients

\begin{tabular}{|lllllll|}
\hline Item & B & S.E. & Sig. & $\operatorname{Exp}(B)$ & \multicolumn{2}{c|}{$95.0 \%$ C.I.for EXP(B) } \\
\cline { 6 - 8 } & & & & & Lower & Upper \\
\hline Crazy paving pattern & 1.082 & 0.417 & 0.010 & 2.952 & 1.303 & 6.689 \\
\hline Diffuse distribution & 1.239 & 0.341 & 0.018 & 2.242 & 1.150 & 4.371 \\
\hline Pericardial distribution & 0.452 & 0.271 & 0.096 & 1.571 & 0.923 & 2.673 \\
\hline CTSS > 12 & 11.494 & 0.270 & 0.038 & 1.090 & 0.643 & 1.849 \\
\hline Pleural effusion & 1.140 & 0.195 & 0.001 & 2.405 & 1.640 & 3.525 \\
\hline Emphysema & 1.179 & 0.499 & 0.018 & 3.250 & 1.222 & 8.646 \\
\hline Pulmonary hypertension & 0.507 & 0.298 & 0.089 & 1.661 & 0.926 & 2.978 \\
\hline
\end{tabular}

Table 4

Multivariable logistic regression model to determine the main CT-based correlates of ICU admission in Covid-19 patients

\begin{tabular}{|lllllll|}
\hline & B & S.E. & Sig. & Exp(B) & \multicolumn{2}{c|}{ 95.0\% C.I.for EXP(B) } \\
\cline { 6 - 8 } & & & & & Lower & Upper \\
\hline Diffuse distribution & -0.126 & 0.326 & 0.699 & 0.882 & 0.465 & 1.670 \\
\hline Pericardial distribution & 0.278 & 0.255 & 0.275 & 1.321 & 0.802 & 2.175 \\
\hline CTSS $>12$ & 1.187 & 0.262 & $<0.001$ & 3.278 & 1.960 & 5.480 \\
\hline Pleural effusion & 1.153 & 0.197 & $<0.001$ & 2.381 & 1.619 & 3.501 \\
\hline Emphysema & 1.740 & 0.432 & $<0.001$ & 5.700 & 2.446 & 13.279 \\
\hline Pulmonary hypertension & 1.175 & 0.297 & $<0.001$ & 3.238 & 1.808 & 5.798 \\
\hline
\end{tabular}

\section{Discussion}

Predicting the severity of COVID-19 is important in several ways. First, based on clinical evidence, laboratory findings as well as imaging findings, it is possible to predict patient mortality as well as the need for their hospitalization or ICU admission, which is the case in the management of patient reception centers, especially when faced with a new wave of disease will be very important and vital. Second, based on this evidence, it is possible to provide scoring systems for the severity and extent of patients' clinical involvement. What has received special attention in recent months is the use of a combination of clinical findings, laboratory markers, and, in addition, the findings of lung CT scan in the design and delivery of such scoring systems. These systems not only make it possible to determine the extent of 
involvement of pulmonary pneumonia, but also make it possible to predict the adverse consequences for patients. In assessing the severity and extent of pulmonary involvement, both the type of lung pathology, the distribution of lesions in the pulmonary lobes, and parenchymal lesions, such as interstitial and vascular lesions, should all be considered. In the present study, we aimed to investigate the relationship between the pattern of involvement and the distribution of lung lesions with the severity of clinical involvement and outcome in patients with COVID-19. What was confirmed in the present study was that, firstly, the distribution and severity of pulmonary involvement in CT patients could be of acceptable diagnostic value for predicting mortality as well as the need for ICU hospitalization (a manifestation of the clinical severity of the disease). Among these, the distribution of lesions in the pulmonary lobes, the presence of some underlying pulmonary complications such as emphysema, pleural effusion or pulmonary hypertension, and the severity of pulmonary involvement based on CT score can play an important role in predicting adverse clinical outcome. In this regard, we first found that the existence of evidence of crazy paving pattern, diffuse distribution as well as pericardial lesions in the lung and higher CT score (mostly higher than 12) as well as the incidence of emphysema and pleural effusion can all increase the risk of death and needing ICU wards. On the other hand, diffuse distribution as well as pericardial lesions, higher CT score, and the appearance of emphysema and pleural effusions or pulmonary hypertension will be important predictors of the need for ICU hospitalization or disease severity that were overall used for introducing a new risk stratification approach. Therefore, in order to provide a new scoring system for risk stratification of patients, it is recommended to use the following criteria and in designing such a system, of course, taking into account other clinical features and other laboratory markers: 1) CTSS score above 12,2) the presence of crazy paving pattern, 3 ) the presence of diffuse or pericardial distribution pattern, 4) the presence of emphysema, pleural effusion or pulmonary hypertension. However, in different studies, there are differences in the factors associated with the prognosis of the disease. For example, the presence of consolidation has been considered as one of the indicators related to the severity of clinical involvement for which we did not find a significant relationship in the present study. Also, different cut points of CTSS have been mentioned in various studies to predict the severity of disease involvement. The observed differences can be due to differences in study design, differences in the experience of specialists in interpreting CT scan findings, differences in patients' underlying clinical conditions, and even geographical differences. In the study of Francone et al, the CT score was higher in patients with more severe involvement of the disease, so that the CT scores higher than 18 was a strong predictor for mortality in patients [11]. It was similar to our study, but their cut-off point was very different from our finding. In a study by Li et al, the presence of consolidation was much higher in the deceased patients than in the living, although in our study there was no difference in this finding. Also, the mean CT score in deceased patients was much higher than in surviving patients, so that a CT score of 14.5 with a sensitivity of $83.3 \%$ and a specificity of $77.3 \%$ was a predictor of mortality in these patients [12] which, of course, was the different cut-off point obtained from our study. However, in the study of Raoufi et al, the CTSS index with a cut-off point of 12 with a sensitivity of $75.8 \%$ and a specificity of $75.7 \%$ was a predictor of patient mortality [13], which, of course, was very close to the cutoff point of our study. Therefore, based on the present study, calculating the CT score along with examining the pattern of involvement (diffuse - pericardial) as well as evidence of emphysema, pleural 
effusion and pulmonary hypertension in scoring system design will be very useful in predicting the clinical severity of COVID-19 disease.

\section{Conclusion}

As a final conclusion, evaluation of the form and pattern of lesion distribution in lung CT has a high value in predicting clinical severity in patients with COVID-19. In this regard, calculating the CT score (score higher than 12) along with observing the pattern of diffuse distribution of lesions and especially associated with other underlying lesions such as emphysema, pleural effusion or pulmonary hypertension can predict patient mortality or will determine the need for hospitalization in the ICU.

\section{Abbreviations}

CT: Computed Tomography, COVID -19: Coronavirus Disease 2019, ICU: Intensive Care Units, CTSS: CT severity score, PCR: Polymerase Chain Reaction, SD: Standard Deviation

\section{Declarations}

\section{Summary statement}

CT scan of the lung in patients with COVID-19 admitted to the ICU is associated with disease severity and mortality.

\section{Authors' contributions}

$\mathrm{KH} \mathrm{N}$ : conceived the original idea, designed the scenarios and approved the final versionthat was submitted, M D, R F and N H: collected the data, M A: carried out the analysis of data. All the authors met the criteria of authorship based on the recommendations of the international commit-tee of medical journal editors.

\section{Funding}

This study was funded by Iran University of Medical Sciences.

\section{Availability of data and materials}

The datasets used and/or analyzed during the current study are available from the corresponding author on reasonable request. Patients or their legal representatives were informed to participate in this study.

\section{Ethics approval and consent to participate}

This study was approved by the Institutional Review Board at the Iran University of Medical Sciences. 
Not applicable.

\section{Competing interests}

The authors declare that they have no competing interests.

\section{References}

1. https://www.santepubliquefrance.fr/maladies-et-traumatismes/maladieset-infectionsrespiratoires/infection-a-coronavirus/articles/infection-aunouveau-coronavirus-sars-cov-2-covid-19france-et-monde\#block-242818.

2. Wu Z, McGoogan JM. Characteristics of and important lessons from the coronavirus disease 2019 (COVID-19) outbreak in China: summary of a report of 72,314 cases from the Chinese Center for Disease Control and Prevention. JAMA. 2020, 323(13):1239-42.

3. Guan W, Ni Z, Hu Y, Liang W, Ou C, He J, et al. Clinical characteristics of coronavirus disease 2019 in China. N Engl J Med. 2020; 382:1708-20.

4. Huang C, Wang Y, Li X. Clinical features of patients infected with 2019 novel coronavirus in Wuhan, China. Lancet. 2020; 395:497.

5. Ai T, Yang Z, Hou H, Zhan C, Chen C, Lv W, et al. Correlation of chest CT and RTPCR testing in coronavirus disease 2019 (COVID-19) in China: a report of 1014 cases. Radiology. 2020 Aug;296(2): E32-E40.

6. Fang $Y$, Zhang H, Xie J, Lin M, Ying L, Pang P, et al. Sensitivity of chest CT for COVID-19: comparison to RT-PCR. Radiology. 2020 Aug;296(2): E115-E117.

7. Pan F, Ye T, Sun P, Gui S, Liang B, Li L, et al. Time course of lung changes at chest CT during recovery from coronavirus disease 2019 (COVID-19). Radiology. 2020;295:715-21.

8. Hani C, Trieu NH, Saab I, Dangeard S, Bennani S, Chassagnon G, et al. COVID-19 pneumonia: a review of typical CT findings and differential diagnosis. Diagn Interv Imagin. 2020; 101:263-8.

9. Revel M-P, Parkar AP, Prosch H, Silva M, Sverzellati N, Gleeson F, et al. COVID-19 patients and the radiology department-advice from the European Society of Radiology (ESR) and the European Society of Thoracic Imaging (ESTI). Eur Radiol. 2020; 30:4903-9.

10. Kunwei Li, Yijie Fang, Wenjuan Li, Cunxue Pan, Peixin Qin, Yinghua Zhong, et al. CT image visual quantitative evaluation and clinical classification of coronavirus disease (COVID-19). Eur Radiol. 2020; 30:4407-16.

11. Marco Francone, Franco lafrate, Giorgio Maria Masci, Simona Coco, Francesco Cilia, Lucia Manganaro, et al. Chest CT score in COVID-19 patients: correlation with disease severity and shortterm prognosis. Eur Radiol. 2020 Jul 4;1-10.

12. Yan Li, Zhenlu Yang, Tao Ai, Shandong Wu, Liming Xia. Association of "initial CT" findings with mortality in older patients with coronavirus disease 2019 (COVID-19). Eur Radiol. 2020 Jun 10;1-8. 
13. Masoomeh Raoufi, Seyed Amir Ahmad Safavi Naini, Zahra Azizan, Fatemeh Jafar Zade, Fatemeh Shojaeian 2, Masoud Ghanbari Boroujeni, et al. Correlation between Chest Computed Tomography Scan Findings and Mortality of COVID-19 Cases; a Cross sectional Study. Arch Acad Emerg Med. 2020 May 14;8(1): e57. eCollection 2020. 